\title{
La declaración electrónica y el título ejecutivo dentro del proceso de cobro coactivo
}

\section{The electronic statement and the enforceable document in the Coercive Collect Jurisdiction}

\author{
DAVid Rueda Mantilla
}

\section{Resumen}

El presente escrito plantea la discusión sobre la posibilidad de que las declaraciones electrónicas presentadas por los contribuyentes constituyan un título ejecutivo con la facultad de prestar mérito ejecutivo. Con ese fin, se repasan los requisitos y las características de los títulos ejecutivos y se analiza si estos se encuentran presentes dentro de las declaraciones electrónicas. El artículo llega a la conclusión de que, desde el punto de vista estrictamente legal, las declaraciones electrónicas por sí solas no constituyen un título ejecutivo con la facultad de prestar mérito ejecutivo. Así mismo, como el Consejo de Estado ha llegado a la conclusión opuesta, se presentan los argumentos traídos de presente en la jurisprudencia y se mencionan las razones por las cuales no son compartidos. Además de lo anterior, este escrito pretende presentar soluciones ante la problemática planteada.

Palabras clave: Declaración electrónica; Título ejecutivo; Proceso de cobro coactivo; Mérito ejecutivo; Documento electrónico.

\begin{abstract}
This paper examines if the electronic statement submitted by tax payers is an enforceable document in the Coercive Collect Jurisdiction. For this purpose, it is analysed if the electronic statement complies with the requirements and general characteristics of the enforceable documents. This paper concludes that the electronic statements do not comply with these requirements and for this reason is not an enforceable document.
\end{abstract}

${ }^{1}$ Abogado (2011), especialista en Gestión Pública e Instituciones Administrativas (2012) y
magíster en Derecho Público para la Función Administrativa (2013) de la Universidad de los
Andes; Master of Science with Merit in Law and Finance: Law and Corporate Finance (2015)
de Queen Mary University of London. Está cursando la especialización en Derecho Tributario
en la Universidad Externado de Colombia. Actualmente se desempeña como abogado en temas
relacionados con Derecho corporativo y Derecho tributario, en Rueda Mantilla Abogados
asociados. Bogotá, Colombia. Correo-e: david@ @uedamantilla.com. Para citar el artículo: Rueda
Mantilla, David. (2016). "La declaración electrónica y el título ejecutivo dentro del proceso de
cobro coactivo", en Revista de Derecho Fiscal n. 9 , Bogotá: Universidad Externado de Colombia.
pp. 77-91. Recibido: 8 de agosto de 2016 . Aprobado: el 20 de septiembre de 2016. DOI: http://
dx.doi.org/10.18601/16926722.n9.06

Revista de Derecho Fiscal.$^{\circ} 8$ • enero-junio de 2016 • pp. 77-91 
However, as soon as the jurisprudence of the High Courts gives a different opinion, this document summarises the arguments given by them and explains why, in our opinion, they are unconvincing. Furthermore, this paper seeks to propose a solution to this situation.

Keywords: Electronic Statement; Enforceable Document; Coercive Collect Jurisdiction; Electronic Document.

Sumario. I. Introducción II. El título ejecutivo y su facultad de prestar mérito ejecutivo 1. Concepto de título ejecutivo 2. Requisitos y características del título ejecutivo 2.1. Requisitos de forma 2.2. Requisitos de fondo 3. El mérito ejecutivo III. El título ejecutivo en el proceso de cobro coactivo IV. La declaración electrónica, por sí sola, no es un título ejecutivo con la facultad de prestar mérito ejecutivo 1. El documento electrónico 2. La declaración electrónica 3. La declaración electrónica no cumple con los requisitos del título ejecutivo 4. La posición del Consejo de Estado y las razones por las cuales no es compartida en este texto V. Propuesta para solventar la problemática 1. Resolver la problemática por intermedio de jurisprudencia 2. Buscar una solución legislativa 3. Ejecutar las declaraciones tributarias con base en el parágrafo del artículo 828 del Estatuto Tributario VI. Conclusiones VII. Bibliografía.

\section{Introducción}

Los procedimientos judiciales y administrativos han sufrido profundos cambios gracias a los avances tecnológicos. Hoy en día, la administración de impuestos permite que los contribuyentes nos relacionemos con ella por intermedio de una gran variedad de servicios electrónicos. Es así como el artículo 579-2 del Estatuto Tributario establece que "el Director de Impuestos y Aduanas Nacionales, mediante resolución, señalará los contribuyentes, responsables o agentes retenedores obligados a cumplir con la presentación de las declaraciones y pagos tributarios a través de medios electrónicos".

La situación anteriormente planteada tiene implicaciones sustanciales dentro del procedimiento tributario. En el presente artículo nos referiremos puntualmente a los alcances que tiene la figura de la "declaración electrónica" dentro de los procesos de cobro coactivo adelantados por la administración de impuestos. El propósito de este texto es demostrar que las declaraciones electrónicas por sí solas no tienen la facultad de prestar mérito ejecutivo, razón por la cual la Administración no debe proferir mandamientos de pago acudiendo a ellas como título ejecutivo. Asimismo, el presente trabajo pretende proponer una solución a la problemática surgida por esta situación.

Con el fin de cumplir con los propósitos planteados en el párrafo anterior, este artículo tendrá la siguiente estructura: en primer lugar, se hará un repaso legal y doctrinario sobre lo que es un título ejecutivo con la facultad de prestar mérito ejecutivo; segundo, se harán unas breves observaciones sobre el título ejecutivo dentro del procedimiento tributario; tercero, se presentarán los argumentos que sustentan la tesis de que una "declaración electrónica" por sí sola no es un título ejecutivo con la facultad de prestar mérito ejecutivo; cuarto, se plantearán las alternativas para resolver esta problemática y se sugerirá cuál de ellas es la más conveniente. Finalmente, a modo de resumen del trabajo, se presentarán unas breves conclusiones. 


\section{El título ejecutivo y su facultad de prestar mérito ejecutivo}

Desde la época de los romanos, en el libro de las instituciones de Justiniano, se ha dicho que "la acción no es otra cosa que el derecho de perseguir en juicio lo que a uno se le debe" (Galindo Vácha, 2006,p. 271). Cuando dicha cosa es cierta e indiscutible, se interpone una acción ejecutiva, mientras que si la misma es incierta y discutible lo procedente es interponer una acción declarativa (Herrera Montañez y Correa Medina, 2012, p. 75), de tal manera que si el acreedor tiene un título ejecutivo nos encontramos frente al primer caso y si carece del mismo estamos en frente del segundo. Por esta razón, para que proceda un proceso ejecutivo o de ejecución es esencial que exista un título ejecutivo (ídem, p. 78).

Ahora bien, con la finalidad de ahondar un poco en lo que es un título ejecutivo, el presente acápite tendrá la siguiente estructura: en primer lugar, se presentará el concepto de título ejecutivo, para luego mencionar sus requisitos y características; finalmente, se harán unas breves precisiones sobre las implicaciones de que un documento preste mérito ejecutivo.

\section{Concepto de título ejecutivo}

Para detallar el título ejecutivo, hay que tener en cuenta que este ha sido definido por diversos autores y pronunciamientos jurisprudenciales. En cuanto a las precisiones ofrecidas por la doctrina, el profesor Hernando Devis Echandía, en un esfuerzo por recopilar las distintas definiciones, conceptualizó el título ejecutivo de la siguiente forma:

Documento o los documentos auténticos que constituyen plena prueba, en el cual o de su- yo conjunto, conste la existencia a favor del demandante y a cargo del demandado, de una obligación expresa, clara y exigible, que además debe ser liquidada si se trata de sumas de dinero, y que reúna los requisitos de origen y forma que exija la ley (1973, p. 478).

Ahora bien, para obtener una definición legal de título ejecutivo es necesario acudir al artículo 488 del Código de Procedimiento Civil, el cual fue replicado posteriormente en el artículo 422 del Código General del Proceso, determinándose allí que el título ejecutivo consiste en un "documento en el cual constare la existencia de una obligación clara, expresa y exigible a cargo de un deudor determinado, en el cual, en cualquier caso, deberá constituir plena prueba en contra de él" (Cermeño, De Bedout, García y Clopatofsky, 2016, p. 253).

Teniendo en cuenta la anterior conceptualización legal y doctrinaria, la jurisprudencia de las altas cortes ha sido unánime en determinar que el título ejecutivo tiene unos requisitos de fondo y otros de forma para prestar mérito ejecutivo (Herrera Montañez y Correa Medina, 2012, p. 88). Los requisitos de forma son dos: i) Que el documento conforme una unidad jurídica y ii) Que sea auténtico y que emane del deudor o de una providencia judicial (Sentencia CE-12-julio-2000). Los requisitos de fondo, por su parte, implican que el documento debe contener "una obligación clara, expresa y exigible y además liquidada o liquidable por simple operación aritmética si se trata de pagar una suma de dinero" (Sentencia CE-12-julio-2000).

\section{Requisitos y características del título ejecutivo}

A continuación, procedo a desarrollar de una forma un poco más profunda los requisitos 
y características anteriormente enunciados del título ejecutivo.

\subsection{Requisitos de forma}

A. Que el documento conforme unidad jurídica

Teniendo en cuenta la complejidad de las relaciones civiles, comerciales y administrativas, "los títulos ejecutivos tienden a estar integrados por documentos plurales" (Mora G., 1980, p. 81). Por esta razón, no es necesario que el título conste materialmente en el mismo documento. En otras palabras, un solo título ejecutivo desde el punto de vista jurídico puede constar en varios documentos desde el punto de vista material (Mora G., 1980, p. 80). Entonces, el requisito de unidad jurídica se refiere a que varios documentos que no constituyen una unidad material, si cumplen con ciertos requisitos y paramentos, pueden constituir un solo título ejecutivo que presta mérito ejecutivo.

B. Que el documento sea auténtico y emane del deudor o de una providencia judicial

Debido a que el título ejecutivo es plena prueba contra el deudor, es necesario que este sea auténtico (Mora G., 1980, p. 83). La autenticidad del título, tal y como lo ha señalado la jurisprudencia nacional, se refiere a que se tenga plena certeza de quién lo suscribió y, en los casos de los títulos valores que son creados por la autonomía de la voluntad, "que el derecho incorporado corresponda al que en su momento exteriorizó el deudor" (Sentencia CE-33586 de 2014).

\subsection{Requisitos de fondo}

A. Existencia de una obligación clara

Tal y como lo señala el profesor Jairo Parra Quijano (1995,p. 265), una obligación “no es clara cuando haya que hacerse explicaciones, deducciones o cualquier otro tipo de rodeos mentales para explicar qué es lo que virtualmente contiene". En este sentido, para que una obligación sea clara es necesario que se den dos supuestos. Por un lado, que se encuentren presentes los elementos esenciales de la obligación: acreedor, deudor, vínculo jurídico y prestación (Herrera Montañez y Correa Medina, 2012, p. 88). Por el otro lado, "que la obligación no genere duda alguna, de modo que sus cantidades y calidades consten de manera específica" (ídem, p. 88). Es importante manifestar que una obligación puede ser determinada o determinable y, por el hecho de ser determinable, no necesariamente pierde su claridad.

\section{B. Existencia de una obligación expresa}

El profesor Hernán Fabio López (1999, p. 388) indica que una obligación es expresa cuando se encuentra señalada inequívocamente en palabras, generalmente escritas. Para Herrera y Correa (2012, p. 89), una obligación es expresa si se encuentra plasmada en el título ejecutivo de manera tal "que de las palabras empleadas en su suscripción no hay puntos oscuros que deben ser escudriñados". Por su parte, Nelson Mora (1980, p. 98) señala que una obligación es expresa cuando en el documento se muestra el "contenido y alcance de la obligación, las partes vinculadas y los términos en que la obligación se ha estipulado". Como se puede 
deducir de las definiciones doctrinarias, que una obligación sea clara o que sea expresa viene a ser prácticamente lo mismo: que no dé lugar a ambigüedades y que estén consagrados todos sus elementos.

\section{Existencia de una obligación exigible}

Que una obligación sea exigible quiere decir que, desde el punto de vista legal, debe ser cumplida de manera inmediata. En palabras de Herrera y Correa (2012, p. 90), "la exigibilidad consiste en que la obligación no penda de condición suspensiva alguna ni de la verificación de plazo o término para manifestar la exigencia de su cumplimiento". Por lo tanto, si una obligación existe, pero aún no se ha cumplido la condición o el plazo para que deba ser cumplida, nos encontraríamos frente a una obligación no exigible.

\section{El mérito ejecutivo}

Un documento presta mérito ejecutivo cuando por intermedio de él se puede exigir el cumplimiento de una obligación acudiendo a un proceso ejecutivo. Como lo señala el profesor Nelson Mora (1980, p. 49), un documento presta mérito ejecutivo cuando cumple con los requisitos necesarios para, por intermedio de un proceso de ejecución, "obligar al deudor al pago o ejecución" de la obligación en él contenida. Entonces, un título ejecutivo solo tiene la facultad de prestar mérito ejecutivo por una sola vez.

Es por lo anterior que el Código de Procedimiento Civil, en el inciso segundo del numeral segundo de su artículo 115, consagraba que, para el caso de las sentencias judiciales, solamente la primera copia prestaría mérito ejecutivo. Por lo tanto, concomitantemente con todos los requisitos enunciados en el presente acápite, para que un título ejecutivo tenga la facultad de prestar mérito ejecutivo es necesario que sea el original y, para el caso de las sentencias judiciales, que sea la primera copia. Es importante resaltar que este requisito de la originalidad se encuentra íntimamente ligado con dos requisitos ya mencionados en este escrito: la autenticidad y la exigencia de que emane del deudor.

\section{EI título ejecutivo en el proceso de cobro coactivo}

Dentro de nuestro ordenamiento jurídico, "encontramos que en ocasiones se les otorga a las autoridades administrativas funciones jurisdiccionales" (Rueda F., 2008, p. 99). Este es el caso de la jurisdicción coactiva, la cual ha sido definida por la jurisprudencia como un "privilegio exorbitante de la Administración, que consiste en la facultad de cobrar directamente, sin que medie intervención judicial, las deudas a su favor, adquiriendo la doble calidad de juez y parte" (Sentencia C-666 de 2000).

Las obligaciones que se pueden cobrar mediante el procedimiento de cobro coactivo, además de ser a favor del Estado, deben ser claras, expresas y exigibles. Por esta razón, el punto de partida de un proceso de cobro coactivo debe ser el título ejecutivo en el que se indiquen el monto y las características de la deuda tributaria (Piza Rodríguez y González Parra, 2010, p. 715). La ley no consagra ninguna característica especial para el título ejecutivo dentro del proceso de cobro coactivo, de tal forma que este debe tener las mismas características y requisitos que tiene cualquier título que preste mérito ejecutivo, a saber, las enunciadas en el primer acápite del presente documento. 
Ahora bien, el artículo 828 del Estatuto Tributario establece cuáles son los documentos que prestan mérito ejecutivo en materia tributaria. Dichos documentos son los siguientes: i) Las liquidaciones privadas contenidas en las declaraciones tributarias presentadas; ii) Las liquidaciones oficiales ejecutoriadas; iii) Los actos de la Administración que fijen sumas líquidas de dinero a favor del fisco; iv) Las garantías y cauciones prestadas a favor de la Nación para afianzar el pago de las obligaciones tributarias, y v) Las sentencias que decidan sobre demandas presentadas en relación con los impuestos, anticipos, retenciones, sanciones e intereses que administra la Dirección General de Impuestos Nacionales.

Siendo el propósito de este artículo el de reflexionar sobre la declaración electrónica y su calidad de título ejecutivo, me enfocaré exclusivamente en el primero de estos documentos que prestan mérito ejecutivo: las liquidaciones privadas y sus correcciones, contenidas en las declaraciones tributarias presentadas. Así pues, el artículo 574 del Estatuto Tributario establece que los contribuyentes, los responsables y los agentes retenedores deben presentar las siguientes declaraciones tributarias:

1. Declaración anual del impuesto sobre la renta y complementarios, cuando de conformidad con las normas vigentes, estén obligados a declarar.

2. Declaración bimestral del impuesto sobre las ventas, para los responsables de este impuesto que pertenezcan al régimen común.

3. Declaración mensual de retenciones en la fuente, para los agentes retenedores del impuesto sobre la renta y complementarios, del impuesto sobre las ventas, y del impuesto de timbre nacional.
4. Declaración del impuesto de timbre, para los documentos y actos sometidos a dicho impuesto.

5. Declaración anual del Impuesto Mínimo Alternativo Simple.

6. Declaración de activos en el exterior.

Teniendo en cuenta lo anterior, una vez estas declaraciones cumplan con los requisitos de los títulos ejecutivos enunciados en el primer acápite de este documento, prestarán mérito ejecutivo para iniciar un procedimiento de cobro coactivo. Además de esto, en caso de que la administración no tenga la forma de allegar la respectiva declaración para iniciar el cobro coactivo, el parágrafo del artículo 828 del Estatuto Tributario establece que "bastará con la certificación del Administrador de Impuestos o su delegado, sobre la existencia y el valor de las liquidaciones privadas oficiales".

\section{La declaración electrónica por sí sola no constituye un título ejecutivo con la facultad de prestar mérito ejecutivo}

Este acápite es el más importante dentro del presente artículo en la medida en que en él se presentan los argumentos para defender la tesis planteada: que la declaración electrónica por sí sola no constituye un título ejecutivo con la facultad de prestar mérito ejecutivo. Para presentar los argumentos en ese sentido, se seguirá con la siguiente estructura: en primer lugar, se hará una breve explicación de lo que es un documento electrónico desde el punto de vista legal y técnico; segundo, desde el punto de vista normativo, se explicará lo que es una declaración electrónica; tercero, teniendo en cuenta los dos puntos anteriores, se mencionarán las razones por las cuales las declaraciones electrónicas, por sí solas, no cumplen 
con los requisitos de los títulos ejecutivos enunciados en el primer acápite del presente documento; cuarto, se hará mención a la jurisprudencia del Consejo de Estado sobre este tema y se explicarán las razones por las cuales la solución dada por este alto tribunal no resulta satisfactoria.

\section{El documento electrónico}

Un documento, tal y como lo señaló el profesor Devis Echandía, es "toda cosa que sirve de prueba histórica indirecta y representativa de un hecho cualquiera" (Rincón Cárdenas, 2006, p. 41). El documento electrónico, por su parte, puede ser definido como "la representación en forma electrónica de hechos jurídicamente relevantes, susceptibles de ser representados en una forma humanamente comprensible" (ídem). Entonces, si se representa un hecho jurídicamente relevante en forma electrónica nos encontramos frente a un documento electrónico.

Los documentos electrónicos "se caracterizan porque sólo pueden ser leídos o conocidos por el hombre gracias a la intervención de sistemas o dispositivos traductores que hacen comprensibles las señales digitales" (Rolero, 2001, p. 3). Por lo tanto, para que se pueda comprender el contenido de un documento electrónico es necesario que contemos con una máquina, generalmente un computador, que traduzca las señales digitales en un lenguaje comprensible para el ser humano. En este sentido, es posible afirmar que un documento electrónico no es "claro" por sí solo sino por la intervención de un dispositivo apropiado que lo traduzca.

Además de lo anterior, como bien lo señala Leonardo Guzmán (1999, p. 122), “en los casos de los documentos electrónicos, el concepto del original que siempre nos remite a la base de papel, desaparece". El documento original no es lo que se ve en la pantalla ni las copias que se puedan imprimir, sino la base magnética modificada por los impulsos electrónicos (ídem). De esta manera, todos los formatos que son visibles al hombre no serían el documento original sino meras copias del mismo. A pesar de que el original del documento electrónico no sea legible, tal y como lo ha señalado la doctrina, no pierde su calidad de documento en la medida en que sigue siendo "una cosa capaz de representar un hecho" (Pinochet Olave, 2002).

Ahora bien, teniendo en cuenta que el original del documento electrónico no es legible pero no por ello pierde su calidad de documento, es necesario estudiar la forma de determinar su valor probatorio y su autenticidad. Esto se puede hacer mediante una tecnología denominada criptografía. "La criptografía es una rama de las matemáticas que, al aplicarse a los mensajes digitales, proporciona las herramientas idóneas" para determinar su autenticidad (Guzmán, 1999, p. 124). Así las cosas, teniendo la necesidad de determinar la autenticidad de los documentos electrónicos, sería conveniente que estos vinieran acompañados de una prueba criptográfica.

Es importante mencionar que con el advenimiento de las nuevas tecnologías el uso del papel cada vez es menor (Rincón Cárdenas, 2006, p. 45). Por lo anterior, y atendiendo a esta realidad, el Congreso de la República profirió la Ley 527 de 1999, "por medio de la cual se define y reglamenta el acceso y uso de los mensajes de datos, de comercio electrónico y de las firmas digitales, y se establecen las entidades de certificación". Esta ley, en su artículo sexto, consagra que "cuando cualquier norma requiera que la información conste por escrito, ese requi- 
sito quedará satisfecho con un mensaje de datos, si la información que este contiene es accesible para su posterior consulta". Por lo tanto, en términos generales, la legislación colombiana les da el mismo valor a los documentos físicos que a los documentos electrónicos.

\section{La declaración electrónica}

El artículo 579-2 del Estatuto Tributario establece que el Director de Impuestos y Aduanas Nacionales "señalará los contribuyentes, responsables o agentes retenedores obligados a cumplir con la presentación de las declaraciones y pagos tributarios a través de medios electrónicos". Con fundamento en esta norma, el Director General de la DIAN profirió la Resolución Número 12761 del 9 de diciembre de 2011, "por la cual se señalan los contribuyentes, responsables, agentes de retención y usuarios aduaneros y demás obligados, que deben presentar las declaraciones y diligenciar los recibos de pago a través de los Servicios Informáticos Electrónicos".

Tal y como lo manifiesta la Administración de Impuestos, cuando se tiene el deber de presentar las declaraciones electrónicas se debe diligenciar el respectivo "formulario directamente sobre la página web" (DIAN, 2015). Este diligenciamiento debe ser realizado atendiendo a lo consagrado en el Decreto 408 de 2001, "por medio del cual se reglamenta el artículo 579-2 del Estatuto Tributario". Para los propósitos del presente trabajo, este decreto trae dos normas que vale la pena mencionar.

La primera es su artículo tercero, el cual establece que "las declaraciones tributarias que se presenten electrónicamente no requieren, para su validez, firma autógrafa de la persona obligada a cumplir con dicho deber formal ni del contador o revisor fiscal". De acuerdo con lo establecido por esta norma, la firma electrónica "se garantizará mediante los certificados y claves secretas de la persona obligada a cumplir con dicho deber formal y del contador o revisor fiscal, que serán expedidos por el sistema”. Esto quiere decir que la plataforma mediante la cual se presentan las declaraciones electrónicas debe contar con unos mecanismos -certificados y claves- idóneos para determinar la autenticidad de la proveniencia de la declaración.

La segunda norma es el artículo 11 del decreto en mención, el cual consagra que "para todos los efectos jurídicos, los documentos electrónicos presentados a través del Sistema de Declaración y Pago Electrónico reemplazarán los documentos físicos en papel". Bajo este entendido, según esta norma, si la declaración en papel es un título ejecutivo con la facultad de prestar mérito ejecutivo, la declaración electrónica también lo sería.

Teniendo en cuenta lo anterior, la administración de impuestos, por lo general, utiliza la información proyectada en la pantalla o en las copias impresas para, con base en ella, iniciar el procedimiento de cobro coactivo. No obstante, es importante tener presente que para poder iniciar este procedimiento es necesario que las declaraciones electrónicas sean títulos ejecutivos con la facultad de prestar mérito ejecutivo y, para ello, no es suficiente con que se equipare el documento electrónico al documento físico, sino que, además, se requiere que cumpla con todos los requisitos de los títulos ejecutivos indicados en la ley. 


\section{La declaración electrónica no cumple con los requisitos del título ejecutivo}

Como ya se mencionó en el primer acápite del presente documento, para que un documento sea un título ejecutivo con la facultad de prestar mérito ejecutivo es necesario que tenga ciertos requisitos y características. A nuestro juicio, la declaración electrónica no cumpliría con dichos requisitos por las razones que se van a exponer a continuación.

Si la administración de impuestos inicia el procedimiento de cobro coactivo valiéndose de la información proyectada en la pantalla o de las copias impresas de la declaración electrónica, tendríamos que estaría utilizando una mera copia del título ejecutivo y no el original del mismo. Esto en cuanto a que, como ya se mencionó en el presente escrito, tratándose de documentos electrónicos, el documento original no es lo que se ve en la pantalla ni las copias que se puedan imprimir sino la base magnética modificada por los impulsos electrónicos (Guzmán, 1999, p. 122).

Es cierto que el artículo 8 de la Ley 527 de 1999 establece que "cuando cualquier norma requiera que la información sea presentada y conservada en su forma original, ese requisito quedará satisfecho con un mensaje de datos". No obstante, las normas de los títulos ejecutivos "no requieren que la información sea presentada y conservada en su forma original" sino que exigen la presencia del documento original para que preste mérito ejecutivo y se pueda ejecutar mediante un proceso de ejecución o un procedimiento de cobro coactivo.

También es cierto que en virtud de lo contemplado en el artículo 11 de la mencionada Ley 527 de 1999, la información contenida en los mensajes de datos tiene pleno valor probatorio. Sin embargo, es importante tener presente que una cosa es que una información tenga valor probatorio y otra muy distinta es que tenga la facultad de prestar mérito ejecutivo. Se insiste en que, de acuerdo con las normas que regulan los títulos ejecutivos, solamente el documento original es el que tiene la facultad de prestar mérito ejecutivo.

Ahora bien, si la administración de impuestos inicia el procedimiento de cobro coactivo valiéndose del documento electrónico original, esto es la base magnética modificada por los impulsos electrónicos, tendríamos que dicho documento, al no poder ser entendido por el hombre sin la intervención de sistemas o dispositivos traductores que lo hagan comprensible, carecería de claridad. En este sentido, no habría una obligación clara en el documento y, por lo tanto, no sería susceptible de prestar mérito ejecutivo.

En resumen, si se entiende que el título ejecutivo son las copias impresas o las proyectadas en la pantalla, carecería de originalidad. Y si se entiende que el título es el documento original que consta en la base magnética modificada por los impulsos electrónicos, carecería de claridad. En ninguno de los dos casos cumpliría con todos los requisitos del título ejecutivo y, por lo tanto, no es susceptible de prestar mérito ejecutivo.

\section{La posición del Consejo de Estado y las razones por las cuales no es compartida en este texto}

Es importante mencionar que el Consejo de Estado ya ha manifestado en varias oportunidades que las declaraciones 
electrónicas son un título ejecutivo con la facultad de prestar mérito ejecutivo (Sentencias CE-16443 de 2009; CE-17155 de 2010). En resumen, son cuatro los argumentos que ha utilizado el máximo tribunal administrativo para defender esta tesis. A continuación, presento cada uno de estos argumentos y explico las razones por las cuales no son compartidos en el presente escrito.

\subsection{Las impresiones de los documentos} electrónicos se reputan auténticas y son prueba de la representación material de las declaraciones tributarias electrónicas.

Con respecto a este argumento presentado por el Consejo de Estado, es importante manifestar que es cierto que las impresiones de las declaraciones electrónicas "se reputan auténticas y son prueba de la representación material de las declaraciones tributarias electrónicas transmitidas a través del sistema informático de la DIAN". No obstante, una cosa es que se reputen auténticas y tengan valor probatorio y otra muy distinta es que tengan la facultad de prestar mérito ejecutivo. Como ya se ha manifestado en el presente escrito, lo que presta mérito ejecutivo, teniendo en cuenta la normatividad de los títulos ejecutivos y el artículo 828 del Estatuto Tributario, son las liquidaciones privadas y sus correcciones, contenidas en las declaraciones tributarias originales o la certificación del administrador de impuestos.

\subsection{El título ejecutivo original está} conformado por la información que reposa en la base de datos del sistema y por el hecho de estar representado en forma de mensaje de datos, el documento no pierde efecto jurídico, validez o fuerza obligatoria.

Es cierto que el título original se encuentra representado en forma de mensaje de datos y que por esta razón no pierde efecto jurídico, validez o fuerza obligatoria. Sin embargo, una cosa es que el documento tenga pleno efecto jurídico, validez y obligatoriedad y otra cosa muy distinta es que sea un título ejecutivo que tenga la facultad de prestar mérito ejecutivo. Para que el documento preste mérito ejecutivo, tal y como ya se ha dicho en reiteradas oportunidades en el presente documento, es necesario que tenga ciertos requisitos y características como lo son, entre otros, la claridad y la originalidad. Para el caso de la declaración electrónica, el documento original, que es el que reposa en la base de datos del sistema, no cumple con el requisito de claridad ya que requiere de un dispositivo traductor para que sea comprensible para el ser humano.

\subsection{Si se exigiera el documento original} para prestar mérito ejecutivo, jamás se podrían ejecutar las declaraciones privadas electrónicas, porque resulta imposible allegar al proceso el original del documento electrónico.

Frente a este argumento presentado por el Consejo de Estado, es importante manifestar que, desde el punto de vista legal, es irrelevante si resulta imposible allegar al proceso el original del documento electrónico para que se puedan ejecutar las obligaciones. Este es un problema de política pública, en la medida en que se podría ver afectado el recaudo, que debe ser resuelto por el legislador y no por las autoridades judiciales. Es importante recordar que a los jueces de la 
república les compete fallar exclusivamente en Derecho y no atendiendo a consideraciones externas a la ley como lo pueda ser la conveniencia en materia de política pública.

Además de lo anterior, la afirmación hecha por el Consejo de Estado no es del todo verídica. Si bien es cierto que en la práctica resulta imposible allegar al proceso el original del documento electrónico, no es cierto que jamás se podrían ejecutar las declaraciones privadas electrónicas. El máximo tribunal administrativo, al hacer esta afirmación, ignora que el parágrafo del artículo 828 del Estatuto Tributario consagra que la certificación del administrador de impuestos presta mérito ejecutivo para los casos de las liquidaciones privadas. En este sentido, atendiendo a lo que establece la ley, las declaraciones privadas electrónicas se pueden ejecutar siempre y cuando el administrador de impuestos profiera un certificado, el cual presta mérito ejecutivo, de la existencia de la respectiva declaración electrónica.

\subsection{El artículo 8 del Decreto 1791 de}

2007 establece que "para todos los efectos jurídicos, las declaraciones presentadas a través de los servicios informáticos electrónicos y los recibos que soportan las transacciones de pago electrónicas remplazaran los documentos físicos en papel". Por lo tanto, las declaraciones presentadas físicamente y las presentadas electrónicamente son documentos que tienen la misma validez jurídica y probatoria.

Es cierto que, de conformidad con lo consagrado por el artículo 8 del Decreto 1797 de 2007 , las declaraciones presentadas a través de los servicios informáticos electrónicos remplazan los documentos físicos de papel y que, por tanto, tienen la misma validez jurídica y probatoria. Por esta razón es que las declaraciones electrónicas son idóneas y conducentes para demostrar la existencia de los títulos ejecutivos. No obstante, una cosa es que dichas declaraciones sirvan como prueba para demostrar que existe el título ejecutivo y otra muy distinta es que sean el título ejecutivo que presta mérito ejecutivo. Como ya se ha dicho insistentemente en el presente documento, para que un documento preste mérito ejecutivo es necesario que tenga unas características y requisitos que las declaraciones electrónicas no tendrían.

\section{Propuesta para solventar la problemática}

Evidentemente, el hecho de que las declaraciones electrónicas no tengan la facultad de prestar mérito ejecutivo resulta inconveniente en la medida en que se dificulta la ejecución de las obligaciones fiscales y, por lo tanto, se podría ver afectado el recaudo. Ante esta situación, hay tres alternativas que vale la pena reflexionar y comentar: i) Resolver la problemática por intermedio de la jurisprudencia, ii) Buscar una solución legislativa y iii) Ejecutar las declaraciones tributarias con base en el parágrafo del artículo 828 del Estatuto Tributario. A continuación, procedo a comentar cada una de estas posibles soluciones.

\section{Resolver la problemática por intermedio de la jurisprudencia}

Como se pudo ver en el acápite anterior, actualmente la problemática planteada está siendo solucionada por intermedio de los pronunciamientos del Consejo de Estado. Esta corporación, haciendo el análisis que 
cuestioné en este artículo, manifestó que las impresiones de las declaraciones electrónicas sí constituyen un título ejecutivo con la facultad de prestar mérito ejecutivo. Esta solución, si bien desde el punto de vista práctico resuelve la indeseable situación, desde el punto de vista jurídico resulta problemática por las razones ya esgrimidas.

Además de lo anterior, se considera que el hecho de que sea la jurisprudencia del Consejo de Estado la que le dé el carácter de título ejecutivo a las declaraciones electrónicas atenta contra el principio de legalidad. Esto en cuanto a que es al poder legislativo y no al judicial al que le corresponde determinar qué documentos tienen la facultad de prestar mérito ejecutivo. En este sentido, se considera que, desde el punto de vista legal y atendiendo al principio de separación y colaboración armónica de poderes, esta solución no es la más adecuada.

\section{Buscar una solución legislativa}

Teniendo en cuenta los argumentos puestos de presente en este artículo, y ante la imperiosa necesidad de adaptar los procesos de ejecución y los procedimientos de cobro coactivo a las circunstancias de la era digital, se considera necesario implementar un mecanismo legal para que los documentos electrónicos cumplan con los requisitos del título ejecutivo y, por tanto, puedan prestar mérito ejecutivo. Así pues, sería preciso que el legislador profiriera una ley en este sentido. Es importante tener presente que no es suficiente con que dicha ley simplemente les dé el carácter de título ejecutivo expresamente a ciertos documentos electrónicos, sino que es necesario encontrar un mecanismo en el que se pueda fusionar la naturaleza del documento electrónico con los elementos esenciales del título ejecutivo.

A mi juicio, son dos los considerandos que esta legislación debe tener en cuenta para darles expresamente el carácter de título ejecutivo a ciertos documentos electrónicos. En primer lugar, se debe determinar puntualmente cuál es el mecanismo tecnológico para que se entienda que se allegó el documento electrónico original y la forma en que este debe ser traducido a un lenguaje que sea comprensible para el ser humano. De esta forma, el título ejecutivo sería el documento electrónico original y es el operador jurídico quien tiene el sistema para traducirlo y comprenderlo. En segundo lugar, es importante que la ley, atendiendo a los avances tecnológicos, busque un mecanismo para que un documento electrónico solo tenga la facultad para prestar mérito ejecutivo por una vez. En otras palabras, que una vez el original de un documento electrónico se haya utilizado para un proceso de ejecución o un procedimiento de cobro coactivo, este quede con una especie de marca de manera tal que no pueda ser usado para prestar mérito ejecutivo por una segunda vez.

\section{Ejecutar las declaraciones tributarias con base en el parágrafo del artículo 828 del Estatuto Tributario}

Como ya se mencionó, el parágrafo del artículo 828 del Estatuto Tributario, para el caso de las declaraciones presentadas, establece que "la certificación del Administrador de Impuestos o su delegado" es un título ejecutivo con la facultad de prestar mérito ejecutivo. Por esta razón, actualmente $-\mathrm{y}$ a pesar de que las declaraciones elec- 
trónicas no constituyan un título ejecutivo con la facultad de prestar mérito ejecutivola administración de impuestos cuenta con las herramientas necesarias para ejecutar, por medio de un procedimiento de cobro coactivo, las obligaciones contenidas en estas declaraciones. En este sentido, y para efectos de ofrecer soluciones al problema planteado en este escrito, no sería necesaria la promulgación de una nueva ley que regule el título ejecutivo electrónico.

Teniendo en cuenta lo anterior, es importante generar conciencia dentro de la administración de impuestos para que los funcionarios antes de iniciar un procedimiento de cobro coactivo, cuando vayan a ejecutar las obligaciones contenidas en una declaración electrónica se aseguren de que el administrador de impuestos emita una certificación de la existencia de la respectiva declaración electrónica. De esta forma, se evitaría iniciar el proceso de cobro coactivo sin contar con el respectivo título ejecutivo, como actualmente viene ocurriendo.

\section{Conclusiones}

Para que proceda un proceso ejecutivo o un procedimiento de cobro coactivo, es indispensable contar con un título ejecutivo que contenga las obligaciones que se pretende ejecutar, el cual debe constar en un documento que cumpla con ciertos requisitos de forma y de fondo. Los requisitos de forma son dos: que el documento conforme una unidad jurídica y que, además de emanar del deudor, sea auténtico. Los requisitos de fondo, por su parte, implican que en el documento debe haber una obligación clara, expresa y exigible. Además de lo anterior, para que el documento preste mérito ejecutivo, es necesario que se trate del título original.
Los documentos que prestan mérito ejecutivo dentro del procedimiento de cobro coactivo por las obligaciones fiscales se encuentran consagrados en el artículo 828 del Estatuto Tributario, encontrándose dentro de estos documentos "las liquidaciones privadas y sus correcciones, contenidas en las declaraciones tributarias presentadas". En virtud del artículo 579-2 del Estatuto Tributario, algunos contribuyentes deben presentar dichas declaraciones a través de los medios electrónicos ofrecidos por la plataforma de la administración de impuestos. Por esta razón, cuando los contribuyentes presentan sus declaraciones por medios electrónicos, la misma no queda en un documento físico sino en un documento electrónico. En este caso, la constancia que tiene la administración de impuestos es una declaración electrónica.

El original de la declaración electrónica, al tratarse de un documento electrónico, no es lo que se ve en la pantalla ni en las copias impresas sino lo que está en la base magnética modificada por los impulsos electrónicos. Dicho documento original no es comprensible para el ser humano sino por intermedio de una máquina idónea que lo traduzca.

Teniendo en cuenta lo explicado en el párrafo anterior, si la administración de impuestos inicia el procedimiento de cobro coactivo valiéndose de la información proyectada en la pantalla o de las copias impresas de la declaración electrónica, tendríamos que estaría utilizando una mera copia del título ejecutivo y no el original del mismo. Si la administración de impuestos inicia el cobro coactivo valiéndose del título electrónico original, en caso de que existiera un mecanismo para allegarlo al proceso, este no sería claro. En cualquiera de los dos casos, el documento no prestaría título ejecutivo: 
en el primer caso por no ser el original y en el segundo, por no estar consagrada la obligación de forma clara.

A pesar de esta realidad, el Consejo de Estado ha sostenido que las declaraciones electrónicas sí constituyen un título ejecutivo con la vocación de prestar mérito ejecutivo. Los argumentos utilizados por esta corporación, como bien se expuso en el último acápite de este artículo, no son lo suficientemente fuertes y, por tanto, pueden ser rebatidos. Por esta razón, es necesario buscar mecanismos para solventar la problemática de que las declaraciones electrónicas no presten mérito ejecutivo.

Dentro de estos mecanismos se propone que el legislador promulgue una ley que regule la materia. Dicha ley debe consagrar que el título ejecutivo es el documento electrónico, el cual es el documento original, y debe contemplar los mecanismos para que pueda ser allegado al proceso y para que el operador jurídico lo traduzca a un lenguaje comprensible para el ser humano. Además de esto, es supremamente importante que dicha ley tenga en cuenta la necesidad de un mecanismo para que un documento electrónico solo tenga la facultad para prestar mérito ejecutivo por una sola vez.

Para finalizar, es importante tener presente que, en este momento, así el legislador no promueva la ley propuesta en el párrafo anterior, es posible ejecutar las obligaciones contenidas en las declaraciones electrónicas. Esto en cuanto a que el parágrafo del artículo 828 del Estatuto Tributario establece que, para el caso de las liquidaciones privadas contenidas en las declaraciones tributarias, servirá como título ejecutivo "la certificación del Administrador de Impuestos o su delegado", sobre la existencia de dichas declaraciones. En este sentido, para ejecutar las declaraciones electrónicas habría título ejecutivo siempre que el administrador de impuestos expidiera la certificación requerida por la ley. 


\section{Bibliografía}

Cermeño C., C. C.; De Bedout, J. C.; García, S. A. y Clopatofsky, C. M. (2016). Procedimiento Tributario. Bogotá: Legis Editores S.A.

Devis Echandía , H. (1973). Compendio de Derecho Procesal Civil. Bogotá: Editorial ABC.

DIAN (6 de noviembre de 2015). DIAN.GOV.CO. Disponible en http://www.dian.gov.co/contenidos/servicios/ presentacion_virtual_preguntas_frecuentes.html.

Galindo Vácha, J. C. (2006). Lecciones de Derecho Procesal Administrativo (segunda ed., Vol. I). Bogotá: Pontificia Universidad Javeriana.

Guzmán, L. (1999). “El documento electrónico", en Revista de la asociación latinoamericana de archivos ALA, 121-131.

Herrera Bravo, R., \& Nuñez, R. A. (1999). Derecho Informatico. Santiago de Chile: Editorial La Ley ltda.

Herrera Montañez, D. A. y Correa Medina, J. A. (2012). El título ejecutivo: Presupuesto de ejecución e instrumento de intimidación al pago. Bogotá: Universidad del Rosario.

López Blanco, H. F. (1999). Instituciones de Derecho procesal civil colombiano. Bogota: Dupre Editores.

Mora G, N. R. (1980). Procesos de ejecución (Vol. I). Bogotá: Temis.

Parra Quijano, J. (1995). Derecho Procesal Civil: Parte Especial. Bogotá: Ediciones Librería el Profesional.

Pinochet Olave, R. (2002). “El documento electrónico y la prueba literal”, en Ius et Praxis, 377-412.

Piza Rodríguez, J. R. y González Parra, O. L. (2010). "La recaudación Tributaria”, en En J. R. Piza Rodríguez, Derecho Tributario, procedimiento y régimen sancionatorio. Bogotá: Universidad Externado de Colombia.

Rincón Cárdenas, E. (2006). Manual de Derecho de comercio electrónico y de internet. Bogotá: Universidad del Rosario.

Rolero, G. L. (2001). "Documento electrónico y firma digital: necesidad de una legislación específica”, en Sistema Argentino de información Jurídica.

Rueda F, M. (2008). Fundamentos de Derecho Procesal Colombiano. Bogotá: Señal Editora.

Vinnio, A. (s.f.). Comentario académico y forense del célebre Arnoldo Vinnio a los cuatro libros de las instituciones imperiales de Justiniano. 